\title{
Nonlinear Output Tracking and Disturbance Rejection for Autonomous Close-Range Rendezvous and Docking of Spacecraft
}

\author{
By Daero LeE, ${ }^{1)}$ Hyochoong BAng, ${ }^{1)}$ Eric A. Butcher ${ }^{2)}$ and Amit K. SANYAL ${ }^{3)}$ \\ ${ }^{1)}$ Department of Aerospace Engineering, Korea Advanced Institute of Science and Technology, Daejeon, Republic of Korea \\ ${ }^{2}$ Department of Aerospace and Mechanical Engineering, University of Arizona, Tucson, AZ, USA \\ ${ }^{3)}$ Department of Mechanical and Aerospace Engineering, New Mexico State University, Las Cruces, NM, USA
}

(Received August 22nd, 2013)

\begin{abstract}
A new control scheme of nonlinear output tracking and disturbance rejection for autonomous close-range rendezvous and docking of spacecraft is proposed in a closed-loop structure. The robustness of the proposed control scheme is achieved through the sum of output-feedback control and state-feedback control as a closed-loop control structure. The designs of both control laws are based on full, 6-degrees-of-freedom, nonlinear dynamic models, which are transformed into a linear-like form using a state dependent Ricatti equation technique. The proposed control scheme ensures closerange rendezvous and meets the state tracking conditions for docking such that the convergence of state tracking errors should be ensured in the presence of unknown disturbances and uncertainties of the system parameters. Compared with the conventional state-dependent Ricatti equation method, the proposed control scheme yields superior state tracking performance and robustness in the presence of external disturbances and uncertain system parameters. The 6-degreesof-freedom numerical simulation results are presented to verify the effectiveness and capability of the proposed control scheme for autonomous close-range rendezvous and docking.
\end{abstract}

Key Words: Nonlinear Output Tracking, Disturbance Rejection, State-Dependent Riccati Equation, Close-Range Rendezvous and Docking

\section{Introduction}

Autonomous rendezvous and docking are important technologies for current and future space programs, including space missions such as supply and repair to the international space station (ISS) and the exploration of the moon, Mars and beyond. A rendezvous mission can be divided into a number of major phases: launch, phasing, far range rendezvous, close-range rendezvous and mating (docking or berthing). ${ }^{1)}$ Each phase has its objectives and end conditions. Rendezvous can also be divided into two main categories: far-range and close-range. Far-range rendezvous occurs after the chaser is inserted into a coplanar orbit with the target. It consists of minimizing the phase and range to less than $10 \mathrm{~km}$ between the chaser and the target. Closerange rendezvous occurs after far-range rendezvous and consists of minimizing the range further to less than $1 \mathrm{~km}$. In general, the phase of close-range rendezvous is divided into two sub-phases: a preparatory phase leading to the final approach corridor, often called "closing" and a final approach phase leading to contact. ${ }^{1)}$ In order to achieve soft and safe docking, extremely delicate and precise position and attitude maneuvers are required. The approach speed of the chaser should be very slow to avoid physical damage with the target. In addition, the tracking errors in relative position, velocity, and relative attitude and angular rate should also be very small. To achieve successful docking, the state tracking errors should be guaranteed consistently

(C) 2014 The Japan Society for Aeronautical and Space Sciences with precise accuracy even in the presence of unknown disturbances.

The chaser spacecraft relative motion in close-range maneuvers is governed by highly nonlinear kinematics and dynamics between the target and chaser spacecraft. Thus, any linear control method is not appropriate for accurate and robust control, especially when large-angle spacecraft maneuvers are required. Various nonlinear control techniques have been developed in the past to address this problem. Among the many control techniques, the state-dependent Riccati equation (SDRE) method emerged as a general design method that provides a systematic and effective means of designing nonlinear controllers, observers and filters in the mid-90s. It has also been widely used for applications in aerospace engineering that require real-time control systems. ${ }^{2-6)}$ The SDRE method is an extension of the linear quadratic regulator (LQR) problem for a linear system. In the SDRE method, nonlinear equations of motion are represented in a linear-like form, called the statedependent coefficient (SDC) form, which corresponds to the system matrix expressed explicitly as a function of the state element. In doing so, the SDRE method partially captures the nonlinearities of the system, transforming the nonlinear system to a non-unique linear structure through the SDC parameterization and minimizing a cost function that has a quadratic-like structure. Most dynamic systems can be directly parameterized in a SDC form. However, there are systems that prevent a direct SDC parameterization and require transformation into the proper structure due to 
the presence of state-independent terms or state-dependent terms that exclude the origin. A systematic and effective design of nonlinear feedback controllers via this method is described in Ref. 5).

Many control problems have used a selected plant model to see the real plant response. They may work successfully using their selected control plant. However, the real plant output may not show stable response when the external disturbances for long-duration missions and the uncertainties of the plant parameters exist. Spacecraft can be influenced by unknown disturbances and uncertain parameters during close-range rendezvous and the docking phase. For instance, atmospheric drag, the Earth oblateness effect and gravitygradient moment can be unknown disturbances when they are not included in the selected plant model to design a controller. The kinematic coupling due to the use of off center of mass (off-CM) points for rendezvous and docking can also be external disturbance. ${ }^{7)}$ As uncertain parameters, there are uncertain mass and moment of inertia. However, Ghartermani et al. proposed a closed-loop control scheme such that the real plant output optimally tracks a given reference state and rejects a given disturbance. ${ }^{6)}$ The solution is formulated based on converting a linear quadratic tracking (LQT) problem into a standard linear quadratic regulation problem where the system equation for the controller design is a time-invariant linear system.

This study was motivated by Ghartermani et al.'s closedloop control scheme to extend from a time-invariant linear system to a time-varying nonlinear system using the SDRE control. This study was also motivated by the need to achieve nonlinear output tracking and disturbance rejection in 6-degrees-of-freedom (6DOF) required for close-range maneuvers and docking. The proposed control scheme does not find and reject unknown external disturbances and uncertain parameters described above, respectively. Instead, it removes the sum of external disturbances including the effect of uncertain parameters in terms of the tracking error between the output from the real plant and the reference state using assumed estimators or precise sensor measurements. Thus, the proposed SDRE-based closed-loop control scheme deals with a robust nonlinear tracking problem in the presence of disturbances instead of a time-invariant linear system for the LQT problem as in Ref. 6). The control scheme consists of nonlinear output tracking and disturbance rejection using the reference states and the tracking error via the real plant. As a result, the control scheme is far more robust than a standard SDRE technique because additional control is summed with a tracking control to cancel the effects of unknown disturbances and uncertain plant parameters. It is then used to design a spacecraft position and attitude controllers such that a chaser spacecraft can perform translational and rotational maneuvers simultaneously. For translational maneuvers, the reference states for closing maneuvers are predetermined using the Clohessy-Wiltshire (CW) guidance scheme. $\left.{ }^{89}\right)$ For rotational maneuvers, the attitude target states including quaternions and angular rates are employed as the reference states to be tracked. Finally, six-degrees of numerical simulation is used to verify the proposed control scheme for close-range rendezvous and docking.

\section{Close-Range Rendezvous and Docking}

A close-range rendezvous is adapted from ESA's Automated Transfer Vehicle (ATV) rendezvous trajectory with respect to the international space station (ISS). ${ }^{10,11)}$ Figure 1 shows close-range rendezvous operations with a V-bar approach of a spacecraft horizontally along the chaser velocity vector to ISS. A closing transfer is to be performed at location $\mathrm{S} 2$ located $1 \mathrm{~km}$ from location $\mathrm{S} 3$, which in turn is $200 \mathrm{~m}$ behind the docking port through a hopping maneuver. After the chaser reaches location S3, a station-keeping maneuver is maintained for a certain time to null out the approach velocity. Then, the final V-bar approach along the docking axis is performed until contact between the target and the chaser is made while the attitude alignment is maintained in the approach corridor. For observability and safety reasons, a cone-shaped approach corridor is defined, within which the approach trajectory has to remain. The cone originates from the docking port of the target and has a half cone angle of 10-15 deg. Such a corridor allows ground operators and/or target crew to assess the accuracy of the approach trajectory via video cameras or other sensor information. If corridor boundaries are violated, stop, retreat or collision avoidance maneuver commands can be issued. The objectives of the closing phase are the reduction of the range to the target and the achievement of conditions allowing the acquisition of the final approach corridor. This means that at the end of this phase the chaser is described by position, velocities, attitudes and angular rates, and is ready to start the final approach on the proper approach axis within the constraints of the safety corridor. The objective of the final approach phase is to achieve docking conditions in terms of positions and velocities and of relative attitude and angular rates. As the chaser comes close to the target, the effects of the thruster plumes on the target vehicle, and disturbance of the attitude and position and overheating of part of the surface and underlying structure can be serious obstacles. In order to minimize these effects for docking, the final contact velocity should be achieved at some distance from the target and will thereafter be kept constant. During the final V-bar approach, the chaser should not penetrate the keep-out sphere to protect the target and achieve the conditions for entering the docking phase. Table 1 presents the rigid mode margins for the worst case of uncertainties for

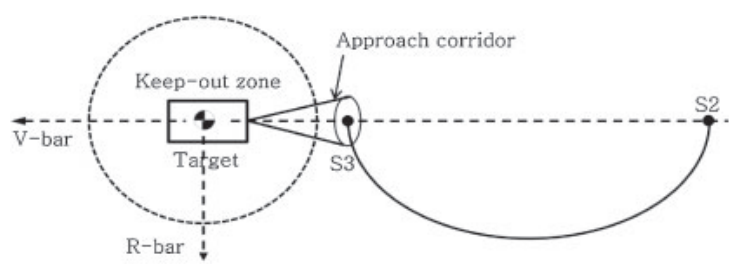

Fig. 1. Close-range rendezvous operations. 
Table 1. Requirements for entering the docking phase $(3 \sigma)$.

\begin{tabular}{lc|lc}
\hline \multicolumn{2}{c|}{ Translational conditions } & \multicolumn{2}{c}{ Rotational conditions } \\
\hline Relative longitudinal closing speed & $0.05-0.10 \mathrm{~m} / \mathrm{s}$ & Misalignment angles & $<5 \mathrm{deg}$ \\
Relative lateral speed & $<0.02 \mathrm{~m} / \mathrm{s}$ & Angular rate (roll) & $<0.40 \mathrm{deg} / \mathrm{s}$ \\
Lateral misalignment & $<0.1 \mathrm{~m}$ & Angular rate (transverse) & $<0.15 \mathrm{deg} / \mathrm{s}$ \\
\hline
\end{tabular}

the attitude and position controllers of the ATV in Refs. 10) and 11). Table 1 also lists the minimum requirements to be achieved for entering the docking phase including navigation and control by $3 \sigma$. This paper employs the minimum requirements for entering the docking phase in Table 1 to verify the capability of the proposed control scheme since it is really used for the ATV docking with the ISS.

\section{Equations of Motions and Problem Formulation}

In this study, we consider a mission for autonomous close-range rendezvous between two spacecraft. The objective of the position control design is to have the chaser track the predefined reference trajectory towards the docking port of the target in the rotating Hill frame $\boldsymbol{H}\left\{\boldsymbol{e}_{r}, \boldsymbol{e}_{t}, \boldsymbol{e}_{n}\right\} .^{8,9,12)}$ The objective of the attitude control design is to have the chaser body-fixed frame $\boldsymbol{B}_{c}\left\{\boldsymbol{b}_{c_{x}}, \boldsymbol{b}_{c_{y}}, \boldsymbol{b}_{c_{z}}\right\}$ aligned with the target body frame $\boldsymbol{B}_{t}\left\{\boldsymbol{b}_{t_{x}}, \boldsymbol{b}_{t_{y}}, \boldsymbol{b}_{t_{z}}\right\}$. The unit vector $\boldsymbol{e}_{r}$ is aligned in a radial direction, the unit vector $\boldsymbol{e}_{n}$ is aligned with the orbit angular momentum, and the unit vector $\boldsymbol{e}_{t}$ is aligned along the direction of the track which completes the triad. The directions of the rotating Hill frame $\boldsymbol{H}\left\{\boldsymbol{e}_{r}, \boldsymbol{e}_{t}, \boldsymbol{e}_{n}\right\}$ are also denoted by R-bar, V-bar and H-bar, respectively, for rendezvous. ${ }^{1)}$ The inertial frame $\boldsymbol{N}\left\{\boldsymbol{n}_{x}, \boldsymbol{n}_{y}, \boldsymbol{n}_{z}\right\}$ is the reference frame for both the rotating Hill frame and two spacecraft body frames. The inertial frame and the Hill frame, as well as the target and chaser body-fixed frames, are illustrated in Fig. 2. The chaser is required to track a predefined trajectory and align its attitude simultaneously.

\subsection{Translational equation of motion}

The relative orbit position vector $\rho$ is expressed in the rotating Hill frame components as $\rho=\left[\begin{array}{lll}x & y & z\end{array}\right]^{\mathrm{T}}$. The inertial target position is expressed through the vector $\boldsymbol{r}_{t}$ while the inertial chaser position is given by $\boldsymbol{r}_{c}$. The subscripts $t$ and $c$ are used to denote the target and chaser, respectively, from now on. The translational motion of the chaser is described by the relative orbital equations of motion. A complete derivation of the relative equations of motion with no disturbances for eccentric orbits is found in Ref. 12). The nonlinear relative equations of motion are given by

$$
\begin{aligned}
& \ddot{x}-2 \dot{f}\left(\dot{y}-y \frac{\dot{r}_{t}}{r_{t}}\right)-x \dot{f}^{2}-\frac{\mu}{r_{t}^{2}}=-\frac{\mu}{r_{c}^{3}}\left(r_{t}+x\right) \\
& \ddot{y}+2 \dot{f}\left(\dot{x}-x \frac{\dot{r}_{t}}{r_{t}}\right)-y \dot{f}^{2}=-\frac{\mu}{r_{c}^{3}} y \\
& \ddot{z}=-\frac{\mu}{r_{c}^{3}} z
\end{aligned}
$$

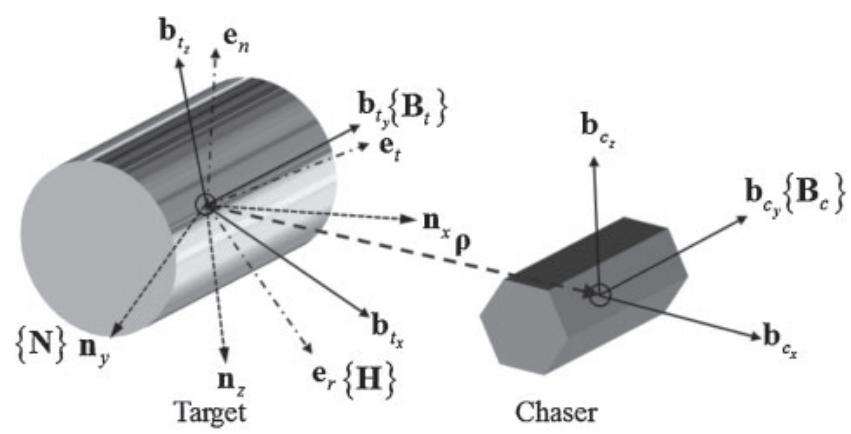

Fig. 2. Target and chaser body-fixed frames, Hill and inertial frames.

where $\mu$ is the gravitational parameter, $f_{t}$ represents the true anomaly of the target, $\dot{f}_{t}$ is true anomaly rate of the target, $r_{t}$ is the orbit radius of the target and $r_{c}=\sqrt{\left(r_{t}+x\right)^{2}+y^{2}+z^{2}}$ is the orbit radius of the chaser. The evolution of the target orbit is governed by the true anomaly acceleration $\ddot{f}_{t}$ and the target orbit radius acceleration $\ddot{r}_{t}$.

$$
\begin{aligned}
& \ddot{f}_{t}=-2 \frac{\dot{r}_{t}}{r_{t}} \dot{f}_{t} \\
& \ddot{r}_{t}=r_{t} \dot{f}^{2}-\frac{\mu}{r_{t}^{2}}
\end{aligned}
$$

When external disturbances are considered, they should be added to Eq. (1). These disturbances include the Earth oblateness $\left(J_{2}\right)$ effect, aerodynamic drag, third body (Sun, Moon, Jupiter, etc.) gravity effects, solar radiation pressure, and thrust applied to the body. In this study, the Earth oblateness $\left(J_{2}\right)$ effect and atmospheric drag are included in the plant by adding them to Eq. (1). The perturbing acceleration due to the $J_{2}$ effect in the Hill frame ${ }^{9)}$ or local-vertical and local-horizontal (LVLH) frame is described as

$$
\begin{aligned}
\boldsymbol{a}_{J_{2}}= & \frac{-3 \mu J_{2} R_{e}^{2}}{r^{4}}\left[\left(\frac{1}{2}-\frac{3 \sin ^{2} i \sin \theta}{2}\right) \boldsymbol{e}_{R}\right. \\
& \left.+\left(\sin ^{2} i \sin \theta \cos \theta\right) \boldsymbol{e}_{T}+(\sin i \sin \theta \cos i) \boldsymbol{e}_{N}\right]
\end{aligned}
$$

where $R_{e}$ is the radius of the Earth, $i$ is the inclination angle and $\theta$ is the argument of latitude. The relative effect of the Earth oblateness due to $J_{2}$ is then

$$
\Delta \boldsymbol{a}_{J_{2}}=\boldsymbol{a}_{J_{2}}\left(r_{c}, i_{c}, \theta_{c}\right)-\boldsymbol{a}_{J_{2}}\left(r_{t}, i_{t}, \theta_{t}\right)
$$

The precise target orbit is assumed to be known from the onboard navigation system of the target. Then, the chaser orbit is computed using the relative orbit with the target orbit. ${ }^{13)}$ The atmospheric drag perturbation ${ }^{14)}$ given by

$$
\boldsymbol{a}_{d}=-\frac{1}{2} \rho\left(\frac{c_{d} A}{m}\right)\|\boldsymbol{v}\| \boldsymbol{v}
$$


is first expressed in the ECI coordinates and is then transformed into the Hill frame using the appropriate direction cosine matrix. Here, $\rho$ is the atmospheric density using the exponential model, while $c_{D}$ is the drag coefficient that depends on the shape and surface. In addition, $A$ is the reference cross-sectional area of the spacecraft, $m$ is the spacecraft mass and $v$ is the spacecraft velocity vector in the ECI coordinates. The relative effect of atmospheric drag, expressed in the Hill frame, is then given by

$$
\begin{aligned}
\Delta \boldsymbol{a}_{d}= & C_{N}^{H}\left(\boldsymbol{a}_{d}\left(\rho_{c}, c_{D_{c}}, A_{c}, m_{c}, \boldsymbol{v}_{c}\right)\right. \\
& \left.-\boldsymbol{a}_{d}\left(\rho_{t}, c_{D_{t}}, A_{t}, m_{t}, \boldsymbol{v}_{t}\right)\right)
\end{aligned}
$$

where $C_{N}^{H}$ is the 3-1-3 rotation sequence $C_{N}^{H}=$ $C_{3}\left(\theta_{t}\right) C_{1}\left(i_{t}\right) C_{3}\left(\Omega_{t}\right)$ for transformation from ECI coordinates to the Hill frame. An unknown and bounded disturbance acceleration $\Delta \boldsymbol{a}_{b}$ between $\pm 10^{-7} \mathrm{~m} / \mathrm{s}^{2}$ is also included in Eq. (1). Then, the sum of all disturbance accelerations is given by

$$
\Delta \boldsymbol{a}=\Delta \boldsymbol{a}_{J_{2}}+\Delta \boldsymbol{a}_{d}+\Delta \boldsymbol{a}_{b}
$$

Thus, the resulting equations of motion including disturbances and control force are used for the translational part of the real plant; i.e.

$\ddot{x}-2 \dot{f}\left(\dot{y}-y \frac{\dot{r}_{t}}{r_{t}}\right)-x \dot{f}^{2}-\frac{\mu}{r_{t}^{2}}=-\frac{\mu}{r_{c}^{3}}\left(r_{t}+x\right)+\frac{F_{x}}{m_{0}}+\Delta a_{x}$

$\ddot{y}+2 \dot{f}\left(\dot{x}-x \frac{\dot{r}_{t}}{r_{t}}\right)-y \dot{f}^{2}=-\frac{\mu}{r_{c}^{3}} y+\frac{F_{y}}{m_{0}}+\Delta a_{y}$

$\ddot{z}=-\frac{\mu z}{r_{c}^{3}}+\frac{F_{z}}{m_{0}}+\Delta a_{z}$

where $\Delta a_{x}, \Delta a_{y}$ and $\Delta a_{z}$ are the components of $\Delta \boldsymbol{a}, F_{x}, F_{y}$ and $F_{z}$ are the applied control force components of $\boldsymbol{F}_{H} \in \mathbb{R}^{3}$ in the Hill frame and $m_{0}$ is the initial chaser's mass. The applied control forces $\boldsymbol{F}_{H}$ can be transformed to the force $\boldsymbol{F}_{c} \in \mathbb{R}^{3}$ in the chaser body-fixed frame using the transformation matrix $T_{H}^{B_{c}}$ from the Hill frame to the chaser body frame; i.e.

$$
\begin{aligned}
& \boldsymbol{F}_{c}=T_{H}^{B_{c}} \boldsymbol{F}_{H} \\
& T_{H}^{B_{c}}=T_{N}^{B_{c}}\left(T_{N}^{H}\right)^{\mathrm{T}}
\end{aligned}
$$

where $T_{N}^{B_{c}}$ is the transformation matrix from the inertial frame to the chaser body frame.

\subsection{Rotational equation of motion}

To fully describe the rotational maneuvers of the rigid body chaser, kinematic and dynamic equations of motion are required. Here, we utilize the Euler parameters (quaternions) for the attitude relative to the inertial frame. The kinematic equation for attitude is

$$
\dot{\boldsymbol{q}}_{c}=\frac{1}{2} \Omega\left(\omega_{c}\right) \boldsymbol{q}_{c}
$$

and the dynamic equation is given by

$$
J_{c} \dot{\boldsymbol{\omega}}_{c}=-\tilde{\boldsymbol{\omega}}_{c} J_{c} \boldsymbol{\omega}_{c}+\boldsymbol{M}_{g}
$$

where $J_{c} \in \mathbb{R}^{3 \times 3}$ is the chaser moment of inertia, $\boldsymbol{\omega}_{c} \in \mathbb{R}^{3}$ is the angular velocity of the chaser and $\boldsymbol{M}_{g} \in \mathbb{R}^{3}$ is the gravity gradient moment, and $\tilde{\boldsymbol{\omega}}_{c}$ and $\Omega\left(\boldsymbol{\omega}_{c}\right)$ are defined respectively by

$$
\tilde{\boldsymbol{\omega}}_{c}=\left[\begin{array}{ccc}
0 & -\omega_{c_{z}} & \omega_{c_{y}} \\
\omega_{c_{z}} & 0 & -\omega_{c_{x}} \\
-\omega_{c_{y}} & \omega_{c_{x}} & 0
\end{array}\right], \quad \Omega\left(\boldsymbol{\omega}_{c}\right)=\left[\begin{array}{cc}
-\tilde{\boldsymbol{\omega}}_{c} & \boldsymbol{\omega}_{c} \\
-\boldsymbol{\omega}_{c}^{\mathrm{T}} & 0
\end{array}\right]
$$

Note that quaternion $\boldsymbol{q}_{c}$ is defined in the chaser's body-fixed frame. The four elements of the quaternion $\boldsymbol{q}_{c}$ are defined by

$$
\begin{aligned}
& q_{c_{i}}=\hat{\boldsymbol{e}} \sin (\vartheta / 2), \quad i=1,2,3 \\
& q_{c_{4}}=\cos (\vartheta / 2)
\end{aligned}
$$

where the unit vector $\hat{\boldsymbol{e}}$ is the axis of rotation and $\vartheta$ is the principal rotation angle. The gravity gradient moment about the body's center of mass is assumed to be known, and given by

$$
\boldsymbol{M}_{g}=3\left(\frac{\mu}{r_{c}^{5}}\right)\left(\left(C_{N}^{B_{c}} \boldsymbol{r}_{c}\right) \times J_{c}\left(C_{N}^{B_{c}} \boldsymbol{r}_{c}\right)\right)
$$

In this study, we consider both disturbance torques or moments for rotational maneuvers and disturbance forces for translational maneuvers. The interaction of the upper atmosphere molecules with the satellite's surface introduces an atmospheric torque $\boldsymbol{T}_{d}$ and is given by

$$
\boldsymbol{T}_{d}=C_{N}^{B_{c}}\left(\boldsymbol{\rho}_{p}-\boldsymbol{\rho}_{m}\right) \times-\frac{1}{2} \rho\left(\frac{c_{D} A}{m}\right)\|\boldsymbol{v}\| \boldsymbol{v}
$$

where $\rho_{p}$ and $\rho_{m}$ are the position vectors of the center of pressure and the center of mass in the inertial frame, respectively. An unknown and bounded disturbance torque $\boldsymbol{T}_{b}$ between $\pm 10 \mathrm{Nm}$ is also included in Eq. (12). Then, the sum of all disturbance torques is given by $\left(\boldsymbol{T}_{d}+\boldsymbol{T}_{b}\right)$. Thus, the resulting rotational dynamics including disturbance torques and control torque $\boldsymbol{\Gamma}_{c}$ is used for the rotational part of the real plant which becomes

$$
\begin{aligned}
\dot{\boldsymbol{q}}_{c} & =\frac{1}{2} \Omega\left(\boldsymbol{\omega}_{c}\right) \boldsymbol{q}_{c} \\
J_{c} \dot{\boldsymbol{\omega}}_{c} & =-\tilde{\boldsymbol{\omega}} J_{c} \boldsymbol{\omega}_{c}+\boldsymbol{M}_{c}+\boldsymbol{\Gamma}_{g}+\left(\boldsymbol{T}_{c}+\boldsymbol{T}_{d}\right)
\end{aligned}
$$

On the other hand, the kinematic and dynamic equations in Eqs. (11) and (12) without considering the effects of unknown disturbances are used for a controller design for rotational maneuvers to evaluate the robustness of the proposed closed-loop control structure.

\section{Summary of the SDRE Method}

Let us consider the autonomous, infinite-horizon, general nonlinear regulator problem for minimizing the cost function

$$
J=\frac{1}{2} \int_{0}^{\infty}\left(\boldsymbol{x}^{\mathrm{T}} Q(\boldsymbol{x}) \boldsymbol{x}+\boldsymbol{u}^{\mathrm{T}} R(\boldsymbol{x}) \boldsymbol{u}\right) \mathrm{d} t
$$

with respect to the state $\boldsymbol{x}$ and control $\boldsymbol{u}$ subject to the nonlinear differential constraints 


$$
\dot{\boldsymbol{x}}=\boldsymbol{f}(\boldsymbol{x})+B(\boldsymbol{x}) \boldsymbol{u}
$$

where $Q(\boldsymbol{x}) \geq 0$ and $R(\boldsymbol{x})>0$ for all $\boldsymbol{x}$ and $\boldsymbol{f}(\mathbf{0})=\mathbf{0}$. The minimization of an infinite-time cost function with a convex integrand that is non-quadratic in $\boldsymbol{x}$ but quadratic in $\boldsymbol{u}$ is considered. The state and input weighting matrices, which are design parameters are assumed to be state-dependent. The details of the SDRE method can be found in Refs. 2), 5) and 15). The SDRE design method consists of the following steps. First, use the direct parameter method to bring Eq. (20) into SDC form, which is a linear-like structure

$$
\begin{aligned}
& \dot{\boldsymbol{x}}=A(\boldsymbol{x}) \boldsymbol{x}+B(\boldsymbol{x}) \boldsymbol{u} \\
& \boldsymbol{f}(\boldsymbol{x})=A(\boldsymbol{x})
\end{aligned}
$$

Although the SDC parameterization is unique in the case of scalar $x$ for all $x \neq 0$, it is not unique in the multivariable case such that the SDC parameterization $A(x)$ itself can be parameterized as $A(\boldsymbol{x}, \alpha)$, where $\alpha$ is a vector of free design parameters. ${ }^{5)}$ The introduction of $\alpha$ creates extra degrees of freedom that are not available in traditional methods. These additional degrees of freedom provided by the non-uniqueness of the SDC parameterization can be used not only to improve controller performance, but also to avoid singularities or loss of controllability, as well as effect tradeoffs between performance, optimality, stability, robustness and disturbance rejection, thus offering a more flexible nonlinear optimal policy. To obtain a valid solution of the SDRE, the pair $\{A(\boldsymbol{x}), B(\boldsymbol{x})\}$ has to be pointwise stabilized in the linear sense so that for all $\boldsymbol{x}$ in the domain of interest, a feasible (i.e., positive definite) solution may be obtained. Second, solve the SDRE

$$
\begin{aligned}
& P(\boldsymbol{x}) A(\boldsymbol{x})+A(\boldsymbol{x})^{\mathrm{T}} P(\boldsymbol{x}) \\
& \quad-P(\boldsymbol{x}) B(\boldsymbol{x}) R(\boldsymbol{x})^{-1} B(\boldsymbol{x})^{\mathrm{T}} P(\boldsymbol{x})+Q(\boldsymbol{x})=0
\end{aligned}
$$

where $P(\boldsymbol{x})$ is state dependent and positive definite for $\boldsymbol{x} \neq \mathbf{0}$. Third, construct the nonlinear feedback controller equation

$$
\boldsymbol{u}(\boldsymbol{x})=-R(\boldsymbol{x})^{-1} B(\boldsymbol{x})^{\mathrm{T}} P(\boldsymbol{x}) \boldsymbol{x}(t)=-K(\boldsymbol{x}) \boldsymbol{x}(t)
$$

where the gain $K(\boldsymbol{x})=R(\boldsymbol{x})^{-1} B(\boldsymbol{x})^{\mathrm{T}} P(\boldsymbol{x})$. In order to perform tracking control, the SDRE controller can be implemented as an integral servomechanism as demonstrated in Stansbery and Cloutier. ${ }^{2,15)}$ This is achieved by augmenting the state vector as follows. First, let us consider the state $\boldsymbol{x}$ decomposed as

$$
\boldsymbol{x}=\left[\begin{array}{ll}
\boldsymbol{x}_{R}^{\mathrm{T}} & \boldsymbol{x}_{N}^{\mathrm{T}}
\end{array}\right]^{\mathrm{T}}
$$

where it is desired for the vector components $\boldsymbol{x}_{R}$ to track a reference command $\boldsymbol{r}_{c}$ and $\boldsymbol{x}_{N}$ is a velocity-like state vector. The state vector $\boldsymbol{x}$ is then augmented with $\boldsymbol{x}_{I}$, the integral state of $\boldsymbol{x}_{R}$

$$
\tilde{\boldsymbol{x}}=\left[\begin{array}{lll}
\boldsymbol{x}_{I}^{\mathrm{T}} & \boldsymbol{x}_{R}^{\mathrm{T}} & \boldsymbol{x}_{N}^{\mathrm{T}}
\end{array}\right]
$$

The augmented system is given by

$$
\dot{\tilde{\boldsymbol{x}}}=\tilde{A}(\tilde{\boldsymbol{x}}, \alpha) \tilde{\boldsymbol{x}}+\tilde{\boldsymbol{B}}(\tilde{\boldsymbol{x}}) \boldsymbol{u}
$$

where

$$
\tilde{A}(\tilde{\boldsymbol{x}}, \alpha)=\left[\begin{array}{cc}
\mathbf{0} & {[I \vdots \mathbf{0}]} \\
\mathbf{0} & A(\boldsymbol{x}, \alpha)
\end{array}\right], \quad \tilde{\boldsymbol{B}}(\boldsymbol{x})=\left[\begin{array}{c}
\mathbf{0} \\
\boldsymbol{B}(\boldsymbol{x})
\end{array}\right]
$$

and the SDRE integral servo controller is given by

$$
\tilde{\boldsymbol{u}}(\tilde{\boldsymbol{x}})=-R(\tilde{\boldsymbol{x}})^{-1} \tilde{\boldsymbol{B}}(\tilde{\boldsymbol{x}})^{\mathrm{T}} \tilde{\boldsymbol{P}}(\tilde{\boldsymbol{x}})\left[\begin{array}{c}
\boldsymbol{x}_{I}-\int \boldsymbol{r}_{c} \mathrm{~d} t \\
\boldsymbol{x}_{R}-\boldsymbol{x}_{c} \\
\boldsymbol{x}_{N}
\end{array}\right]
$$

Recall that in order for the SDRE to have a solution, the pointwise detectability condition must be satisfied. This is achieved by penalizing the integral states with the corresponding nonzero diagonal elements of $\tilde{Q}(\tilde{\boldsymbol{x}})$. In this study, an alternative SDRE tracking approach is used to design a tracking controller in Ref. 16), which does not require increasing the state dimension.

\section{Proposed Closed-Loop Structure}

In this paper, we propose a SDRE-based closed-loop structure to ensure that the reference state is achieved in the presence of disturbances by rejecting the disturbances completely. Figure 3 shows a proposed nonlinear closedloop control structure. It consists of a SDRE-based outputfeedback controller (OFC) plus a SDRE-based state-feedback controller (SFC). The OFC is designed using a SDRE tracking method to ensure that the reference state $\boldsymbol{x}_{r}(t)$ is tracked. The cost function in Eq. (17) is used to design the SDRE-based OFC.

The nonlinear system equation of the OFC is described by

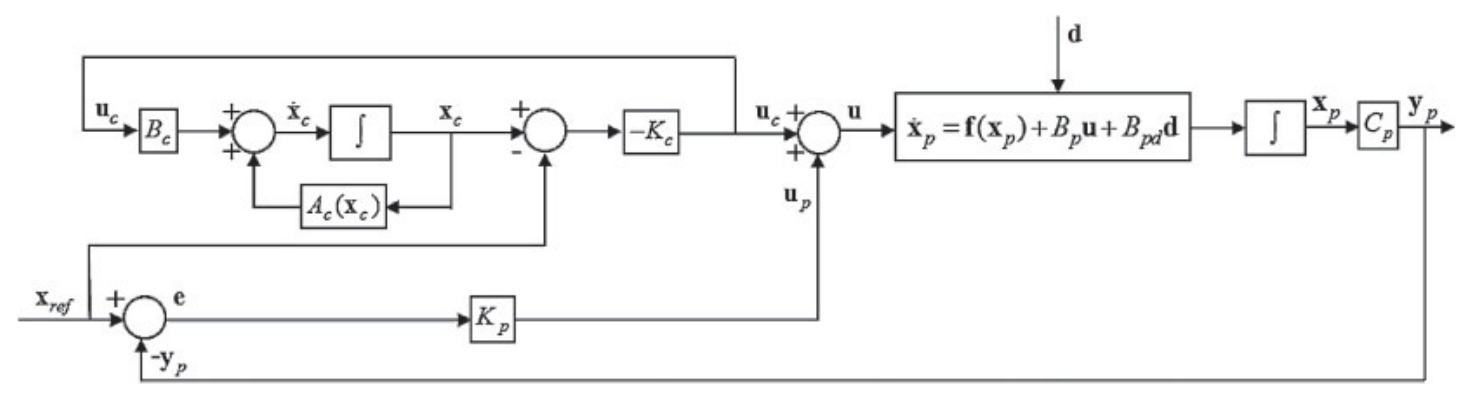

Fig. 3. Proposed nonlinear closed-loop control structure. 


$$
\dot{\boldsymbol{x}}_{o}=\boldsymbol{f}\left(\boldsymbol{x}_{o}\right)+B_{o} \boldsymbol{u}_{o}=A_{o}\left(\boldsymbol{x}_{o}\right) \boldsymbol{x}_{o}+B_{o} \boldsymbol{u}\left(\boldsymbol{x}_{o}\right)
$$

where $\boldsymbol{x}_{o}$ is the $n$-dimensional OFC state vector. The nonlinear system equation of the SFC is employed as the real plant that shows the real control response and is described by

$$
\begin{aligned}
\dot{\boldsymbol{x}}_{p} & =\boldsymbol{f}\left(\boldsymbol{x}_{p}\right)+B_{p} \boldsymbol{u}+B_{p d} \boldsymbol{d} \\
& =A_{p}\left(\boldsymbol{x}_{p}\right) \boldsymbol{x}_{p}+B_{p} \boldsymbol{u}+B_{p d} \boldsymbol{d}, \\
\boldsymbol{y}_{p} & =C_{p} \boldsymbol{x}_{p}
\end{aligned}
$$

where $\boldsymbol{x}_{p}$ is the $n$-dimensional SFC state vector, $\boldsymbol{d}$ is the unknown disturbance signal, $\boldsymbol{y}_{p}$ is the output from the real plant, and $C_{p}$ is the $6 \times 6$ identity matrix.

The nonlinear equations, $\boldsymbol{f}\left(\boldsymbol{x}_{o}\right)$ and $\boldsymbol{f}\left(\boldsymbol{x}_{p}\right)$ are identical to each other and they are transformed into $A_{o}\left(\boldsymbol{x}_{o}\right) \boldsymbol{x}_{o}$ and $A\left(\boldsymbol{x}_{p}\right) \boldsymbol{x}_{p}$, respectively via the SDC parameterizations. The nonlinear equation $\boldsymbol{f}\left(\boldsymbol{x}_{o}\right)$ in Eq. (28) is used to design the OFC $\boldsymbol{u}_{o}$ using the SDRE tracking method whereas the nonlinear system of the SFC in Eq. (29) is used as the real plant to compute real system response using the control $\boldsymbol{u}$. If the OFC $\boldsymbol{u}_{o}$ is fed into the real plant with unknown disturbances included, the real plant state $\boldsymbol{x}_{p}$ may not track the reference state $\boldsymbol{x}_{r}$ accurately because of disturbances which are not considered in designing the OFC. Thus, the tracking error $\boldsymbol{e}=\boldsymbol{x}_{r}-\boldsymbol{y}_{p}$ does not converge to zero in the steady state. However, the reference state can be tracked accurately once the SDRE tracking control $\boldsymbol{u}_{o}$ is replaced with $\boldsymbol{u}$ where the additional SFC control $\boldsymbol{u}_{p}$ is summed with the OFC control $\boldsymbol{u}_{o}$ to make up for the real plant tracking error $\boldsymbol{e}$. If we define an augmented state

$$
\tilde{\boldsymbol{x}}_{p o}=\left[\begin{array}{ll}
\boldsymbol{x}_{p}^{\mathrm{T}} & \boldsymbol{x}_{o}^{\mathrm{T}}
\end{array}\right]^{\mathrm{T}}
$$

the nonlinear closed-loop system is given by

$$
\begin{aligned}
\dot{\tilde{\boldsymbol{x}}}_{p o} & =A\left(\tilde{\boldsymbol{x}}_{p o}\right) \tilde{\boldsymbol{x}}_{p o}+B \boldsymbol{u}+B_{p d} \boldsymbol{d}, \\
\boldsymbol{y}_{p} & =C_{p} \boldsymbol{x}_{p}
\end{aligned}
$$

where

$$
\begin{aligned}
& A\left(\tilde{\boldsymbol{x}}_{p o}\right)=\left(\begin{array}{cc}
A_{p}\left(\boldsymbol{x}_{p}\right) & 0_{6 \times 6} \\
0_{6 \times 6} & A_{o}\left(\boldsymbol{x}_{o}\right)
\end{array}\right), \\
& B=\left(\begin{array}{c}
B_{p} \\
B_{o}
\end{array}\right), \quad B_{d}=\left(\begin{array}{c}
B_{p d} \\
0_{6 \times 3}
\end{array}\right)
\end{aligned}
$$

The following two assumptions are made in order to design a closed-loop control structure that can reject disturbances.

Assumption 1: The state-space representation of Eq. (30) is stabilizable and detectable.

Assumption 2: The real plant output $\boldsymbol{y}_{p}$ can be measured or estimated using precise sensors.

If the disturbance signal $\boldsymbol{d}$ is known, it is possible to design a SDRE tracking controller using the nonlinear system equation in Eq. (29). In addition, it is not necessary to consider Eqs. (28) and (29) separately to design a tracking controller. The SDRE tracking controller only is then able to track the reference states in the presence of known disturbances. However, this study considers the opposite condition that there are unknown disturbances when the OFC is designed. The SDRE-based OFC may only track the reference states in the presence of unknown disturbances. Thus, an additional feedback control is required to reject unknown disturbances. Because the output from the real plant can be measured or estimated, the precise tracking error can be computed. The tracking error $\boldsymbol{e}$ is the difference of the output from the real plant and the reference state $\boldsymbol{x}_{r}$. The additional control can be generated by multiplying the control gain $K_{p}$ with the tracking error $\boldsymbol{e}$. As a result, the new control law is the sum of OFC $\boldsymbol{u}_{o}$ and SFC $\boldsymbol{u}_{p}$ that rejects unknown disturbances while the states track the reference states as follows.

$$
\begin{aligned}
\boldsymbol{u} & =\boldsymbol{u}_{o}+\boldsymbol{u}_{p} \\
\boldsymbol{u}_{o} & =-R\left(\boldsymbol{x}_{o}\right)^{-1} B^{\mathrm{T}}\left(\boldsymbol{x}_{o}\right) P(\boldsymbol{x})\left(\boldsymbol{x}_{o}-\boldsymbol{x}_{r}\right), \\
\boldsymbol{u}_{p} & =R^{-1}\left(\boldsymbol{x}_{p}\right) B^{\mathrm{T}}\left(\boldsymbol{x}_{p}\right) P\left(\boldsymbol{x}_{p}\right) \boldsymbol{e}
\end{aligned}
$$

where the OFC gain is $K_{o}\left(\boldsymbol{x}_{o}\right)=R^{-1}\left(\boldsymbol{x}_{o}\right)^{-1} B^{\mathrm{T}}\left(\boldsymbol{x}_{o}\right) P(\boldsymbol{x})$ and the SFC gain $K_{p}\left(\boldsymbol{x}_{p}\right)=R\left(\boldsymbol{x}_{p}\right)^{-1} B^{\mathrm{T}}\left(\boldsymbol{x}_{p}\right) P\left(\boldsymbol{x}_{p}\right)$. The numerical values for both the gains become identical when the disturbances are completely rejected. Unlike the SDRE integral servo controller in Eq. (27), the new control law does not need to increase the state dimension because the additional control effort is added to reject disturbances.

\section{Reference State}

The hopping maneuvers in the close-range rendezvous are predetermined using the analytical solution of the Clohessy-Wiltshire (CW) equation state transition matrix (STM) ${ }^{8,9)}$ An intercept velocity can be computed using the STM of the CW equation from any given initial position. The reference states then can be propagated to the desired position. According to the flight time, the intercept velocity and the shape of the hopping maneuvers are varied. When the flight time is long enough, the approach speed becomes slow. If a relative state vector is defined as

$$
\boldsymbol{x}_{r}(t)=\left[\begin{array}{c}
\boldsymbol{\rho}_{r} \\
\dot{\boldsymbol{\rho}}_{r}
\end{array}\right]=\left[\begin{array}{llllll}
x_{r} & y_{r} & z_{r} & \dot{x}_{r} & \dot{y}_{r} & \dot{z}_{r}
\end{array}\right]^{\mathrm{T}}
$$

then, the relative position and velocity are propagated using the STM as

$$
\left[\begin{array}{l}
\boldsymbol{\rho}_{r}(t) \\
\dot{\boldsymbol{\rho}}_{r}(t)
\end{array}\right]=\Phi\left(t, t_{0}\right)\left[\begin{array}{l}
\boldsymbol{\rho}_{r}\left(t_{0}\right) \\
\dot{\boldsymbol{\rho}}_{r}\left(t_{0}\right)
\end{array}\right]
$$

where 


$$
\Phi\left(t, t_{0}\right)=\left[\begin{array}{ccc}
4-3 \cos n t & 0 & 0 \\
6(\sin n t-n t) & 1 & 0 \\
0 & 0 & \cos n t \\
3 n \sin n t & 0 & 0 \\
-6 n(1-\cos n t) & 0 & 0 \\
0 & 0 & -n \sin n t
\end{array}\right.
$$

is the state transition matrix obtained from the $\mathrm{CW}$ equations and $n$ is the mean motion of the target. The state transition matrix $\Phi\left(t, t_{0}\right)$ is partitioned into four $3 \times 3$ partitions as

$$
\Phi\left(t, t_{0}\right)=\left[\begin{array}{cc}
M(t) & N(t) \\
S(t) & T(t)
\end{array}\right]
$$

The necessary initial relative velocity to intercept the target at the final time is then obtained as

$$
\dot{\boldsymbol{\rho}}_{r}\left(t_{0}\right)=N\left(t_{f}\right)\left(\boldsymbol{r}_{d}\left(t_{f}\right)-M\left(t_{f}\right) \boldsymbol{\rho}_{r}\left(t_{0}\right)\right)
$$

where $\boldsymbol{r}_{d}\left(t_{f}\right)$ is the desired final position, $t_{0}$ is the initial time and $t_{f}$ is the final flight time. The reference positions and velocities are obtained to intercept the desired final position $\boldsymbol{r}_{d}\left(t_{f}\right)$ by propagating the initial reference state from the initial time to the final flight time. After the closing transfer reaches location S3 in Fig. 1, station-keeping is then performed for a few minutes to null out the approach velocity and prepare for the next phase. Finally, a straight line V-bar maneuver is then performed until the condition for the docking phase is achieved. While the station-keeping maneuver is performed, the chaser is required to be bounded in three-dimensional space to maintain the desired constant position and zero velocity. After the station-keeping phases, straight-line forced-motion trajectories are used for the V-bar final approaches with constant velocity from location S3 to the docking port. This type of trajectory implements a constant relative velocity of $V_{x}=\left(x_{r}(t)-x_{r}\left(t_{0}\right)\right) / t$ with respect to the target between the initial $x_{r}\left(t_{0}\right)$ and the desired $x_{r}(t)$, with the approach velocity along the target maintained at zero. The equation for the reference $x_{r}(t)$ along the V-bar direction is simply given by

$$
x_{r}(t)=x_{r}\left(t_{0}\right)+v_{x} t
$$

where $x_{r}(t)$ at the terminal time becomes the desired location. In order to carry out attitude alignment between the target and the chaser, the attitude and angular velocity of the target are required. In this study, the attitude information of the target is assumed to be available via on-board navigation. Then, the solutions of numerical integrations for the kinematics and dynamics of the target in Eqs. (9) and (10) become the attitude states of the target. Thus, the reference state $\boldsymbol{x}_{r}$ for translational and rotational maneuvers is used for the controller design in Eq. (31) and is given by

$$
\boldsymbol{x}_{r}=\left[\begin{array}{llll}
\boldsymbol{\rho}_{r}^{\mathrm{T}} & \dot{\boldsymbol{\rho}}_{r}^{\mathrm{T}} & \boldsymbol{q}_{t}^{\mathrm{T}} & \boldsymbol{\omega}_{t}^{\mathrm{T}}
\end{array}\right]^{\mathrm{T}}, \quad \boldsymbol{x}_{r} \in \mathbb{R}^{9} \times \mathbb{S}^{3}
$$

$$
\left.\begin{array}{ccc}
\frac{\sin n t}{n} & \frac{2}{n}(1-\cos n t) & 0 \\
-\frac{2}{n}(1-\cos n t) & \frac{4 \sin n t-3 n t}{n} & 0 \\
0 & 0 & \frac{\sin n t}{n} \\
\cos n t & 2 \sin n t & 0 \\
-2 \sin n t & 4 \cos n t-3 & 0 \\
0 & 0 & \cos n t
\end{array}\right]
$$

$\left.\begin{array}{c}0 \\ 0 \\ \frac{\sin n t}{n} \\ 0 \\ 0 \\ \cos n t\end{array}\right]$

\section{Proposed Closed-Loop Controller Design for Trans- lational and Rotational Maneuvers}

In this study, we consider an example of close-range rendezvous performing three hopping maneuvers, one stationkeeping, a straight line approach as well as attitude maneuver. The objectives of the controller design are to have the chaser at the relative position and velocity $\rho$ and $\dot{\rho}$, and track the reference relative position and velocity $\boldsymbol{\rho}_{r}$ and $\dot{\boldsymbol{\rho}}_{r}$ at a certain distance from the target, which is located at the origin of the Hill frame, in the presence of disturbances. It is also desired to have the chaser spacecraft body frame $\boldsymbol{B}_{c}\left\{\boldsymbol{b}_{c_{x}}, \boldsymbol{b}_{c_{y}}, \boldsymbol{b}_{c_{z}}\right\}$ align with the target body frame $\boldsymbol{B}_{t}\left\{\boldsymbol{b}_{t_{x}}, \boldsymbol{b}_{t_{y}}, \boldsymbol{b}_{t_{z}}\right\}$ in the presence of disturbances. The state and control variables for this problem are chosen to be

State variables:

$$
\boldsymbol{x}=\left[\begin{array}{llll}
\boldsymbol{\rho}^{\mathrm{T}} & \dot{\boldsymbol{\rho}}^{\mathrm{T}} & \boldsymbol{q}_{c}^{\mathrm{T}} & \boldsymbol{\omega}_{c}^{\mathrm{T}}
\end{array}\right]^{\mathrm{T}}, \quad \boldsymbol{x} \in \mathbb{R}^{9} \times \mathbb{S}^{3}
$$

Control variables:

$$
\boldsymbol{u}_{o}=\left[\begin{array}{ll}
\boldsymbol{F}_{H}^{\mathrm{T}} & \boldsymbol{\Gamma}_{c}^{\mathrm{T}}
\end{array}\right]^{\mathrm{T}}, \quad \boldsymbol{u}_{o} \in \mathbb{R}^{6}
$$

The nonlinear equations of motions to design a controller for both translational and rotational maneuvers is written in state space form using Eqs. (1), (11) and (12), and is given by

$$
\begin{aligned}
& \dot{\boldsymbol{x}}=\left[\begin{array}{c}
\dot{\boldsymbol{\rho}} \\
\ddot{\boldsymbol{\rho}} \\
\dot{\boldsymbol{q}}_{c} \\
\dot{\boldsymbol{\omega}}_{c}
\end{array}\right]=\left[\begin{array}{c}
\dot{\boldsymbol{x}} \\
\dot{y} \\
\dot{z} \\
2 \dot{f}\left(\dot{y}-y \frac{\dot{r}_{t}}{r_{t}}\right)+x \dot{f}^{2}+\frac{\mu}{r_{t}^{2}}-\frac{\mu}{r_{c}^{3}}\left(r_{t}+x\right) \\
-2 \dot{f}\left(\dot{x}-x \frac{\dot{r}_{t}}{r_{t}}\right)+y \dot{f}^{2}-\frac{\mu}{r_{c}^{3}} y \\
-\frac{\mu}{r_{c}^{3}} z \\
\frac{1}{2} \Omega\left(\boldsymbol{\omega}_{c}\right) \boldsymbol{q}_{c} \\
J_{c}^{-1}\left(-\tilde{\boldsymbol{\omega}}_{c} J_{c} \boldsymbol{\omega}_{c}+\boldsymbol{M}_{g}\right)
\end{array}\right] \\
& +\left[\begin{array}{cc}
0_{3 \times 3} & 0_{3 \times 3} \\
\frac{1}{m_{0}} \boldsymbol{I}_{3 \times 3} & 0_{3 \times 3} \\
0_{4 \times 3} & 0_{4 \times 3} \\
0_{3 \times 3} & J_{c}^{-1}
\end{array}\right]\left[\begin{array}{c}
\boldsymbol{F}_{H} \\
\boldsymbol{\Gamma}_{c}
\end{array}\right]
\end{aligned}
$$


However, there is some modification for the chaser quaternion kinematics in Eq. (11). A small constant $\varepsilon_{\omega}=-0.0001$ is added to the chaser quaternion kinematics in order to avoid uncontrollability in the SDC parameterization because the chaser spacecraft body rates are unstable. ${ }^{2,3)}$ Instead of the original quaternion kinematics in Eq. (11), the following modified quaternion kinematics is used for the controller design.

$$
\dot{\boldsymbol{q}}_{c}=\frac{1}{2}\left[\begin{array}{l}
\varepsilon_{\omega} q_{c_{1}}+q_{c_{4}} \omega_{c_{x}}-q_{c_{3}} \omega_{c_{y}}+q_{c_{2}} \omega_{c_{z}} \\
\varepsilon_{\omega} q_{c_{2}}+q_{c_{3}} \omega_{c_{x}}+q_{c_{4}} \omega_{c_{y}}-q_{c_{1}} \omega_{c_{z}} \\
\varepsilon_{\omega} q_{c_{3}}-q_{c_{2}} \omega_{c_{x}}+q_{c_{1}} \omega_{c_{y}}+q_{c_{4}} \omega_{c_{z}} \\
\varepsilon_{\omega} q_{c_{4}}-q_{c_{1}} \omega_{c_{x}}-q_{c_{2}} \omega_{c_{y}}-q_{c_{3}} \omega_{c_{z}}
\end{array}\right]
$$

The particular $A(\boldsymbol{x})$ selected for this application is given by

$$
A(\boldsymbol{x})=\left[\begin{array}{ccccccccc}
0_{3 \times 3} & 0_{3 \times 3} & 0_{3 \times 1} & 0_{3 \times 1} & 0_{3 \times 1} & 0_{3 \times 1} & 0_{3 \times 1} & 0_{3 \times 1} & 0_{3 \times 1} \\
a_{4: 6 \times 1: 3} & a_{4: 6 \times 4: 6} & 0_{3 \times 1} & 0_{3 \times 1} & 0_{3 \times 1} & 0_{3 \times 1} & 0_{3 \times 1} & 0_{3 \times 1} & 0_{3 \times 1} \\
0_{1 \times 3} & 0_{1 \times 3} & \frac{\varepsilon_{\omega}}{2} & 0 & 0 & 0 & \frac{q_{c_{4}}}{2} & -\frac{q_{c_{3}}}{2} & \frac{q_{c_{2}}}{2} \\
0_{1 \times 3} & 0_{1 \times 3} & 0 & \frac{\varepsilon_{\omega}}{2} & 0 & 0 & \frac{q_{c_{3}}}{2} & \frac{q_{c_{4}}}{2} & \frac{-q_{c_{1}}}{2} \\
0_{1 \times 3} & 0_{1 \times 3} & 0 & 0 & \frac{\varepsilon_{\omega}}{2} & 0 & \frac{-q_{c_{2}}}{2} & \frac{q_{c_{1}}}{2} & \frac{q_{c_{4}}}{2} \\
0_{1 \times 3} & 0_{1 \times 3} & 0 & 0 & 0 & \frac{\varepsilon_{\omega}}{2} & \frac{-q_{c_{1}}}{2} & \frac{-q_{c_{2}}}{2} & \frac{-q_{c_{3}}}{2} \\
0_{1 \times 3} & 0_{1 \times 3} & \frac{a_{11,7} q_{c_{1}}}{\left(\boldsymbol{q}_{s}^{\mathrm{T}} \boldsymbol{q}_{s}\right)} & \frac{a_{11,7} q_{c_{2}}}{\left(\boldsymbol{q}_{s}^{\mathrm{T}} \boldsymbol{q}_{s}\right)} & \frac{a_{11,7} q_{c_{3}}}{\left(\boldsymbol{q}_{s}^{\mathrm{T}} \boldsymbol{q}_{s}\right)} & \frac{a_{11,7} q_{c_{4}}}{\left(\boldsymbol{q}_{s}^{\mathrm{T}} \boldsymbol{q}_{s}\right)} & 0 & 0 & 0 \\
0_{1 \times 3} & 0_{1 \times 3} & \frac{a_{12,7} q_{c_{1}}}{\left(\boldsymbol{q}_{s}^{\mathrm{T}} \boldsymbol{q}_{s}\right)} & \frac{a_{12,7} q_{c_{2}}}{\left(\boldsymbol{q}_{s}^{\mathrm{T}} \boldsymbol{q}_{s}\right)} & \frac{a_{12,7} q_{c_{3}}}{\left(\boldsymbol{q}_{s}^{\mathrm{T}} \boldsymbol{q}_{s}\right)} & \frac{a_{12,7} q_{c_{4}}}{\left(\boldsymbol{q}_{s}^{\mathrm{T}} \boldsymbol{q}_{s}\right)} & 0 & 0 & 0 \\
0_{1 \times 3} & 0_{1 \times 3} & \frac{a_{13,7} q_{c_{1}}}{\left(\boldsymbol{q}_{s}^{\mathrm{T}} \boldsymbol{q}_{s}\right)} & \frac{a_{13,7} q_{c_{2}}}{\left(\boldsymbol{q}_{s}^{\mathrm{T}} \boldsymbol{q}_{s}\right)} & \frac{a_{12,7} q_{c_{3}}}{\left(\boldsymbol{q}_{s}^{\mathrm{T}} \boldsymbol{q}_{s}\right)} & \frac{a_{12,7} q_{c_{4}}}{\left(\boldsymbol{q}_{s}^{\mathrm{T}} \boldsymbol{q}_{s}\right)} & 0 & 0 & 0
\end{array}\right]
$$

where

$$
\begin{aligned}
& a_{4: 6 \times 1: 3}=\left[\begin{array}{ccc}
\left(\dot{f}^{2}-\frac{\mu}{r_{c}^{3}}\right) & \left(-2 \dot{f}^{\frac{r_{t}}{r_{t}}}\right) & 0 \\
\left(2 \dot{f}^{\frac{r_{t}}{r_{t}}}\right) & \left(\dot{f}^{2}-\frac{\mu}{r_{c}^{3}}\right) & 0 \\
0 & 0 & \left(-\frac{\mu}{r_{c}^{3}}\right)
\end{array}\right] \\
& a_{4: 6 \times 4: 6}=\left[\begin{array}{ccc}
\left(\left(\frac{\mu}{r_{t}^{2}}-\frac{\mu}{r_{c}^{3}} r_{t}\right) \dot{x} /\left(\dot{\boldsymbol{\rho}}^{\mathrm{T}} \dot{\boldsymbol{\rho}}\right)\right) & \left(\left(\frac{\mu}{r_{t}^{2}}-\frac{\mu}{r_{c}^{3}} r_{t}\right) \dot{y} /\left(\dot{\boldsymbol{\rho}}^{\mathrm{T}} \dot{\boldsymbol{\rho}}\right)\right) & \left(\left(\frac{\mu}{r_{t}^{2}}-\frac{\mu}{r_{c}^{3}} r_{t}\right) \dot{z} /\left(\dot{\boldsymbol{\rho}}^{\mathrm{T}} \dot{\boldsymbol{\rho}}\right)\right) \\
-2 \dot{f}+\left(\left(\frac{\mu}{r_{t}^{2}}-\frac{\mu}{r_{c}^{3}} r_{t}\right) \dot{x} /\left(\dot{\boldsymbol{\rho}}^{\mathrm{T}} \dot{\boldsymbol{\rho}}\right)\right) & \left(\left(\frac{\mu}{r_{t}^{2}}-\frac{\mu}{r_{c}^{3}} r_{t}\right) \dot{y} /\left(\dot{\boldsymbol{\rho}}^{\mathrm{T}} \dot{\boldsymbol{\rho}}\right)\right) & \left(\left(\frac{\mu}{r_{t}^{2}}-\frac{\mu}{r_{c}^{3}} r_{t}\right) \dot{z} /\left(\dot{\boldsymbol{\rho}}^{\mathrm{T}} \dot{\boldsymbol{\rho}}\right)\right) \\
\left(\left(\frac{\mu}{r_{t}^{2}}-\frac{\mu}{r_{c}^{3}} r_{t}\right) \dot{x} /\left(\dot{\boldsymbol{\rho}}^{\mathrm{T}} \dot{\boldsymbol{\rho}}\right)\right) & \left(\left(\frac{\mu}{r_{t}^{2}}-\frac{\mu}{r_{c}^{3}} r_{t}\right) \dot{y} /\left(\dot{\boldsymbol{\rho}}^{\mathrm{T}} \dot{\boldsymbol{\rho}}\right)\right) & \left(\left(\frac{\mu}{r_{t}^{2}}-\frac{\mu}{r_{c}^{3}} r_{t}\right) \dot{z} /\left(\dot{\boldsymbol{\rho}}^{\mathrm{T}} \dot{\boldsymbol{\rho}}\right)\right)
\end{array}\right] \\
& {\left[\begin{array}{lll}
a_{11,7} & a_{12,7} & a_{13,7}
\end{array}\right]^{\mathrm{T}}=J_{c}^{-1}\left(-\tilde{\boldsymbol{\omega}}_{c} J_{c} \boldsymbol{\omega}_{c}+\boldsymbol{M}_{g}\right)}
\end{aligned}
$$

When the nonlinear equations of motions in Eqs. (41) and (42) are factored into a linear-like structure via SDC parameterization, there is a violation due to the presence of stateindependent terms or bias terms in Eqs. (41) and (42). Using the property that the speed of the chaser $\|\dot{\rho}\|$ never becomes zero, these can be multiplied and divided by the squared magnitude of the velocity even though any component of the spacecraft velocity $\boldsymbol{v}$ can become zero. This bias term $b(t)$ can then be factored as $b(t)=\left[b(t) \boldsymbol{v}^{\mathrm{T}} /\left(\boldsymbol{v}^{\mathrm{T}} \boldsymbol{v}\right)\right] \boldsymbol{v} .^{6,15)}$ The chaser rotational dynamics given in Eq. (41) cannot be directly factored into a linear form because of the first term, though there is no bias term. However, the rotational dynamics given in Eq. (41) can be factored into a linear form using the property that the square of the quaternion never becomes zero, thus leading to $b(t)=\left[b(t) \boldsymbol{q}^{\mathrm{T}} /\left(\boldsymbol{q}^{\mathrm{T}} \boldsymbol{q}\right)\right] \boldsymbol{q}$. As a result, the singularity in the SDC parameterization is avoided and the system matrix $A(\boldsymbol{x})$ is obtained. The satisfying state weighting matrix and the control weighting matrix for the cost function used in Eq. (19) are given respectively by 


$$
\begin{aligned}
& Q(\boldsymbol{x})=\left[\begin{array}{cccc}
10^{4} I_{3 \times 3} & 0_{3 \times 3} & 0_{3 \times 4} & 0_{3 \times 3} \\
0_{3 \times 3} & 0_{3 \times 3} & 0_{3 \times 4} & 0_{3 \times 3} \\
0_{4 \times 3} & 0_{4 \times 3} & 10^{3} I_{4 \times 4} & 0_{4 \times 3} \\
0_{3 \times 3} & 0_{3 \times 3} & 0_{3 \times 4} & 0_{3 \times 3}
\end{array}\right] \\
& R(\boldsymbol{x})=\left[\begin{array}{cc}
I_{3 \times 3} & 0_{3 \times 3} \\
0_{3 \times 3} & 10^{-4} I_{3 \times 3}
\end{array}\right]
\end{aligned}
$$

The weighting matrices can be adjusted during the control tracking in order to achieve tighter tracking control results while avoiding an initial high control effort, which may result in actuator saturation. The control using the SDREbased closed-loop control structure is then computed using Eq. (31) once control gains $K_{o}$ and $K_{p}$ are evaluated with the OFC state $\boldsymbol{x}_{o}$ and the output of the SFC $\boldsymbol{y}_{p}$.

\section{Numerical Results and Analysis}

A 6DOF numerical simulation of a chaser and a target is created for a close-range rendezvous to demonstrate the performance of the proposed closed-loop control in the presence of external disturbances and uncertain system parameters. The chaser is required to perform a close-range rendezvous that consists of closing transfer, station-keeping and final approach maneuver as well as large angle change and complicated position change. We label the target and chaser as the ISS and ATV, respectively. The chaser is required to perform a close-range rendezvous that consists of a closing transfer, a station-keeping and final approach maneuver as well as large angle change. The chaser is initially located at a position of $\left[\begin{array}{llll}1 & -1000 & 1\end{array}\right]^{\mathrm{T}} \mathrm{m}$ with zero relative velocity in the Hill frame. In order to show the effectiveness of the controller, the initial reference position of $\left[\begin{array}{lll}0 & -1000 & 0\end{array}\right]^{\mathrm{T}} \mathrm{m}$ and zero relative velocity in the Hill frame are selected. A closing transfer is executed to transfer from a point $\mathrm{S} 2,980 \mathrm{~m}$ behind the docking port to a point $\mathrm{S} 3,230 \mathrm{~m}$ behind the docking port. The docking port is assumed to be located at $\left[\begin{array}{lll}0 & -20 & 0\end{array}\right]^{\mathrm{T}} \mathrm{m}$ in the Hill frame. The initial attitude of the chaser is rotated by 120 degrees of roll, -50 degrees pitch, and 20 degrees of yaw in a 3-2-1 rotation sequence with respect to the target body frame while the body frame of the target is assumed to coincide with the Hill frame. The corresponding chaser quaternion is then given by $\boldsymbol{q}_{c}\left(t_{0}\right)=\left[\begin{array}{llll}0.4391 & -0.0718 & 0.8097 & 0.3827\end{array}\right]^{\mathrm{T}}$. The target maintains the Earth pointing orientation whose angular rate is always constant while a close-range rendezvous is performed. The initial mass of the chaser $m_{0}$ is assumed as the ATV's launch mass of $19,600 \mathrm{~kg}$. The moment of inertias of the target and chaser are given by $J_{t}$ and $J_{c}$ in Eq. (46). ${ }^{1,17)}$ Thus, the target and chaser are $y$ and $z$ axes-symmetric, respectively.

$$
J_{t}=10^{6} \times\left[\begin{array}{ccc}
128 & 0 & 0 \\
0 & 107 & 0 \\
0 & 0 & 201
\end{array}\right] \quad \mathrm{kg} \mathrm{m}^{2}
$$

$$
J_{c}=10^{6} \times\left[\begin{array}{ccc}
0.59 & 0 & 0 \\
0 & 1.38 & 0 \\
0 & 0 & 1.38
\end{array}\right] \mathrm{kg} \mathrm{m}^{2}
$$

The reference cross-sectional areas of the target and chaser $A_{c}$ in Eq. (7) are given by $72.8 \times 108.5 \mathrm{~m}^{2}$ and $4 \times 8.4 \mathrm{~m}^{2}$. The length and diameter of the chaser are $9.03 \mathrm{~m}$ and $4.48 \mathrm{~m}$, respectively. The drag coefficients and air densities of the target and chaser in Eq. (7) are identically given by $c_{D_{t}}=c_{D_{c}}=2.0$ and $\rho_{t}=\rho_{c}=4.0 \times 10^{-13} \mathrm{~kg} / \mathrm{m}^{3}$, respectively. The chaser is treated as a rigid body which considers translational and rotational maneuvers simultaneously. Both the translation and rotation of the chaser is described about the center of mass (CM). The relative translational and rotational dynamics is described about CM points of the target and chaser, respectively. The quaternions and angular rates of the target are generated using Eqs. (11) and (12) after replacing the subscript $t$ with $c$. The constant angular velocity of $\omega_{t}(t)=\left[\begin{array}{lll}0 & 0.0011 & 0\end{array}\right]^{\mathrm{T}} \mathrm{rad} / \mathrm{s}$ which corresponds to the Earth-pointing spacecraft is also used. The initial angular velocity of the chaser spacecraft is given by $\omega_{c}(0)=$ $\left[\begin{array}{lll}0 & 0 & 0\end{array}\right]^{\mathrm{T}}$. Thus, the chaser has to perform a large angle maneuver as well as a complicated position change during close-range rendezvous. The target is assumed to have the following orbital elements listed in Table 2.

Figures 4 and 5 show disturbance accelerations on the chaser due to the Earth oblateness $J_{2}$ and atmospheric drag between the target and the chaser in the Hill frame. The disturbance acceleration due to the Earth oblateness $J_{2}$ decreases to zero as the chaser approaches the target. On the other hand, the along-track component of disturbance acceleration due to atmospheric drag is dominantly bounded. These perturbations are considered as unknown disturbances that are not included in the system equations to design a controller for translational maneuver. The unknown bounded disturbance accelerations between $\pm 10^{-6} \mathrm{~m} / \mathrm{s}^{2}$ are also generated. The disturbance due the Earth oblateness is the most dominant among the disturbance accelerations. All of these disturbance accelerations are then summed and substituted into the real plant as shown in Fig. 3.

Figures 6 and 7 show the Earth gravity gradient moment and the aerodynamic torque on the chaser. Unlike unknown disturbances, the gravity gradient moment is known and included in the chaser rotational dynamics. The known Earth gravity moment disturbance is much larger than the known aerodynamic torque disturbance. Unknown bounded disturbance torques between $\pm 10 \mathrm{Nm}$ are also generated.

Table 2. Orbital elements of the target.

\begin{tabular}{lc}
\hline Semimajor axis & $6739.188 \mathrm{~km}$ \\
Eccentricity & 0.0005817 \\
Inclination & $51.64 \mathrm{deg}$ \\
Right ascension of ascending node & $316.44 \mathrm{deg}$ \\
Argument of perigee & $40.90 \mathrm{deg}$ \\
Mean anomaly & $319.24 \mathrm{deg}$ \\
\hline
\end{tabular}




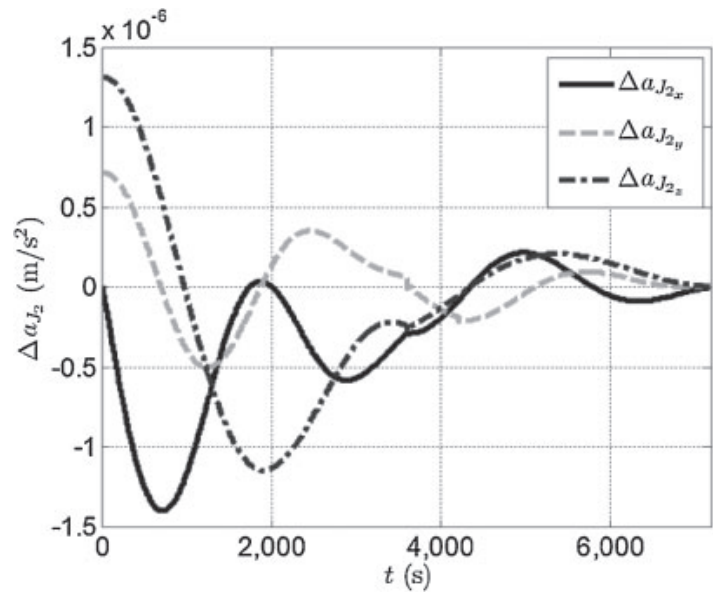

Fig. 4. Disturbance due to the Earth oblateness.

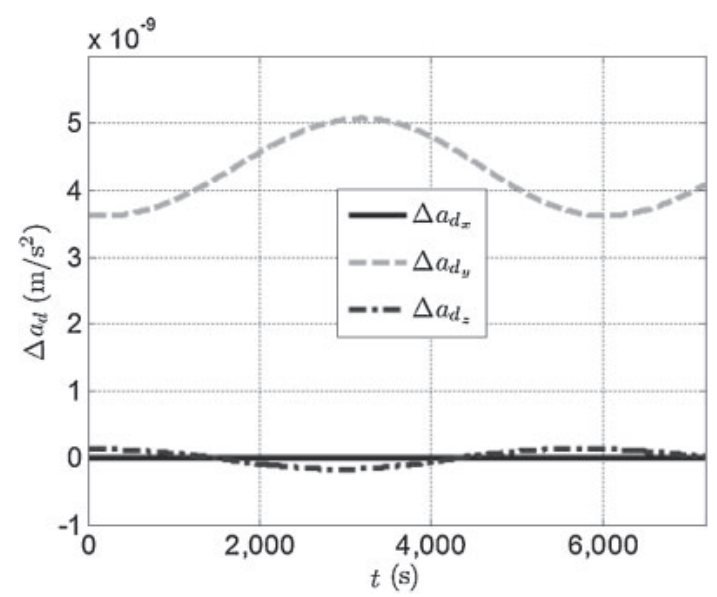

Fig. 5. Disturbance due to atmospheric drag.

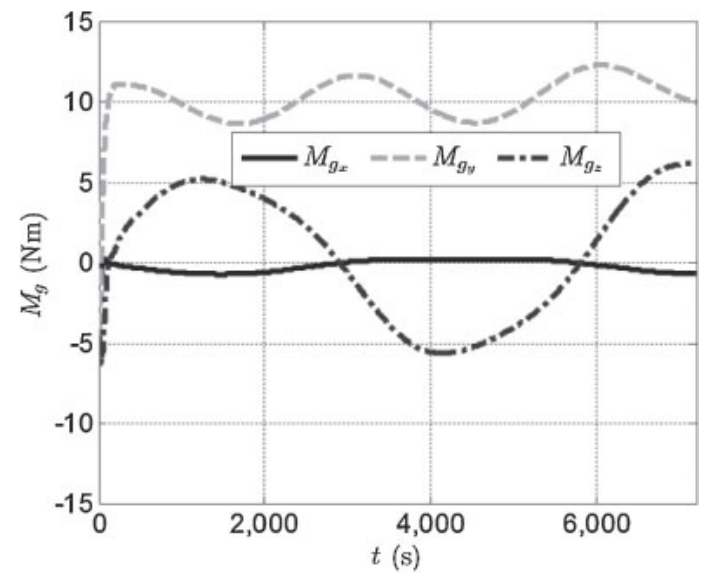

Fig. 6. Earth gravity-gradient moment.

The unknown disturbance moment or torque are also summed and substituted into the real plant as shown in Fig. 3. In addition, the uncertain system parameters such as mass varying $m(t)$ and moment of inertia of the chaser $J_{c}$ are used to test the robustness of the proposed controller. Originally, the initial chaser mass should decrease as the propellant is consumed. However, the initial mass of $m_{0}=19,600 \mathrm{~kg}$ is used for the control distribution matrix $B_{o}$ in Eq. (28). In order to test the robustness of the proposed

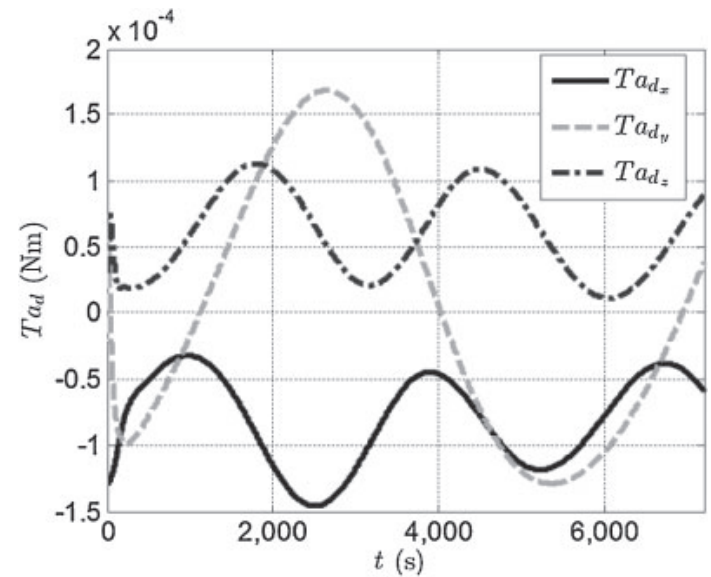

Fig. 7. Atmospheric torque.

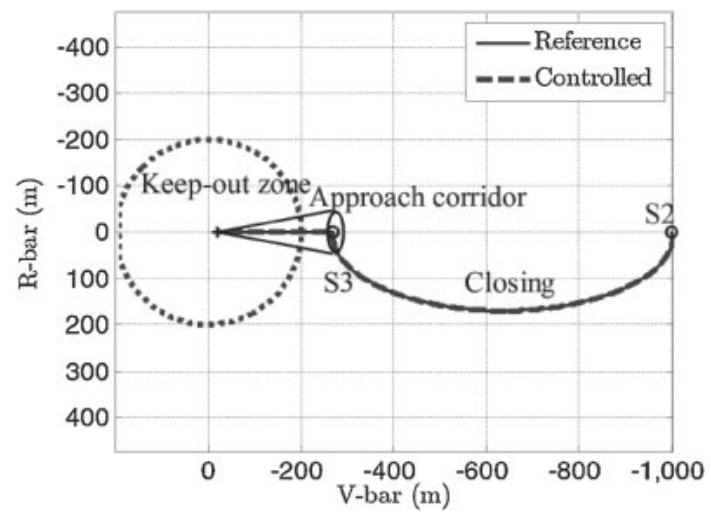

Fig. 8. Planar close-range rendezvous trajectory.

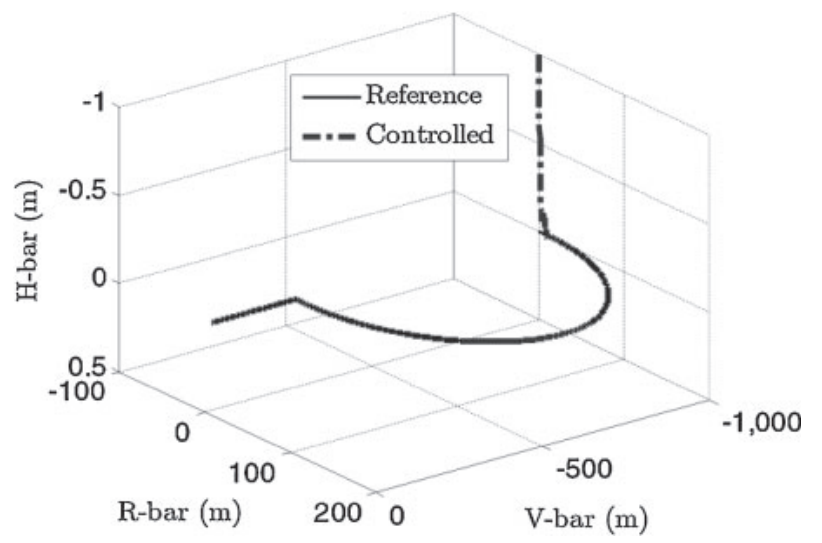

Fig. 9. Three-dimensional close-range rendezvous trajectory.

control in the translational maneuver and rotational maneuver, the chaser's initial mass and moments of inertia are replaced with $0.9 m_{0}=17,640 \mathrm{~kg}$ which is assumed to be $10 \%$ decreased and $1.1 J_{c}$ which is assumed to be $10 \%$ increased, respectively. Then, they are used for building up the control distribution matrix $B_{p}$ in Eq. (29), respectively. 8.1. Translational maneuver result

Planar and three-dimensional close-range rendezvous trajectories are presented in Figs. 8 and 9, respectively. The reference closing transfer trajectory is propagated using the CW state transition matrix in Eqs. (34) and (35), and 


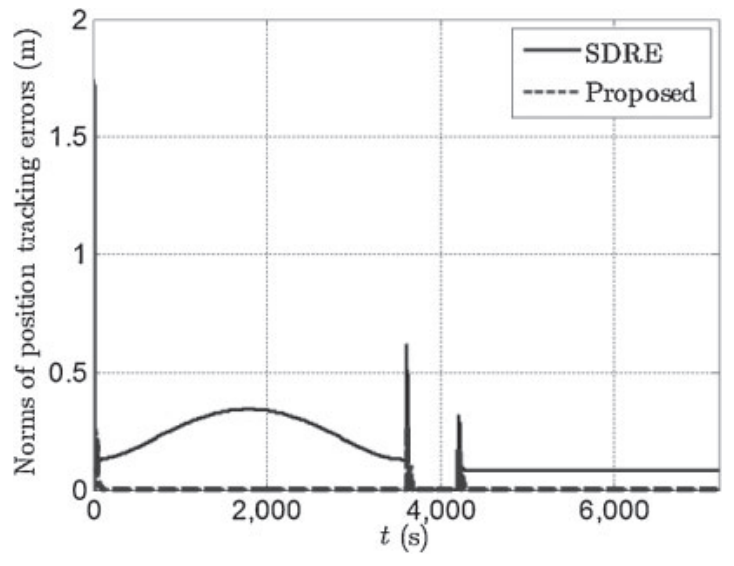

Fig. 10. Norms of position tracking errors.

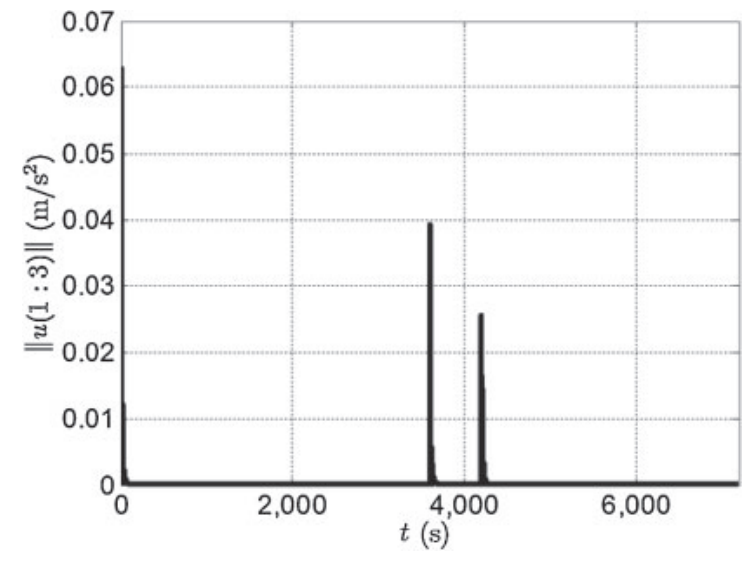

Fig. 11. Norm of total control acceleration.

the initial relative velocity to intercept the target at the final time in Eq. (36). From there on, a final translational maneuver along the V-bar direction is performed until contact is made between the target and chaser docking mechanisms. The target is surrounded by $200 \mathrm{~m}$ spherical area called keep-out zone where no vehicle shall penetrate this volume within the approach corridor.

Figure 8 shows that the chaser enters \pm 10 degrees of the approach corridor after station-keeping at S3 and passes through it without crossing the keep-out zone. Figure 10 shows the norms of position tracking errors using the SDRE control or output feedback control $\boldsymbol{u}_{o}(1: 3)$ and the proposed control scheme $\boldsymbol{u}(1: 3)$. The norm of position tracking error using the proposed control scheme converges to zero in the presence of unknown external disturbances and uncertain chaser's mass. It also satisfies the requirement for lateral misalignment in Table 1 except for S2 where station-keeping is performed from S3. On the other hand, the norm of position tracking error using the SDRE control only does not fully converge to zero from S3 because of unknown external disturbances and uncertain chaser's mass. The temporarily increased norms of position tracking errors using the proposed control scheme after S2 can be acceptable because they are caused by the intercept velocity due to the closing transfer and the final translational maneuver after station-keeping at S3, respectively. Figure 11 shows

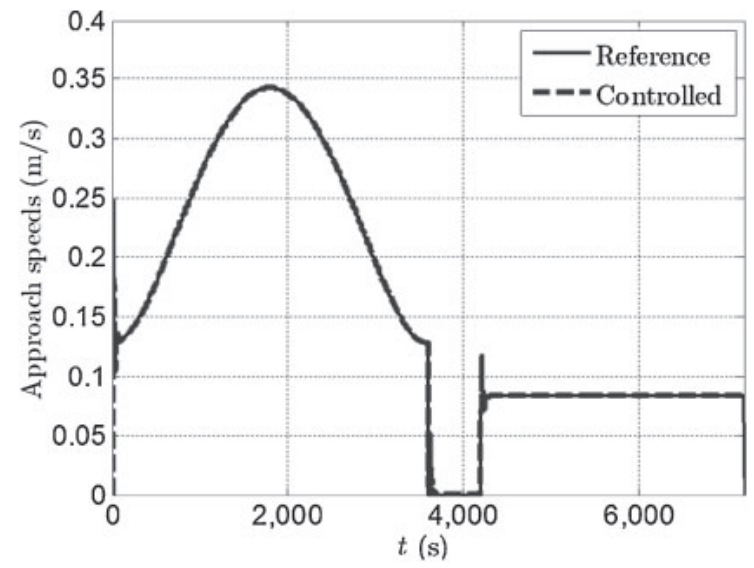

Fig. 12. Approach speed of the chaser.

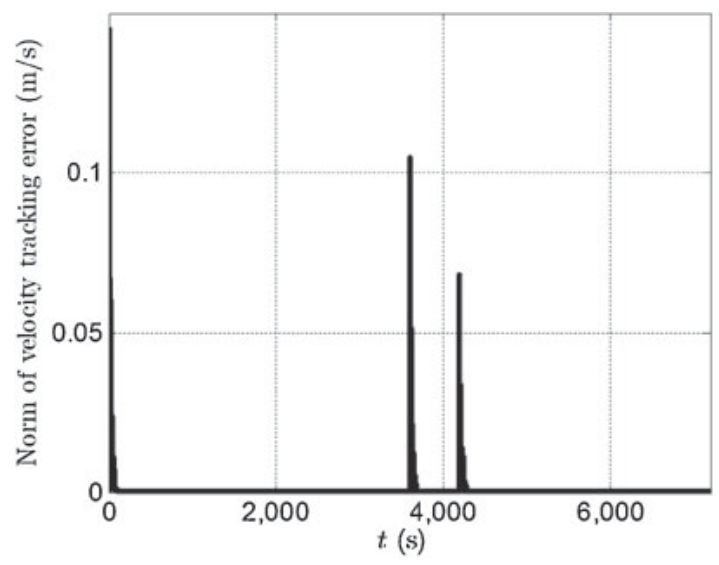

Fig. 13. Norm of velocity tracking error.

the norm of control acceleration using the proposed control scheme. It increases temporarily at S2 and S3 to execute the closing transfer and final translational maneuver.

Figure 12 presents the approach speeds of the chaser and reference, respectively. The approach speed of the chaser increases almost up to $0.343 \mathrm{~m} / \mathrm{s}$ during closing transfer, decreases to zero during station-keeping and converges to $0.833 \mathrm{~m} / \mathrm{s}$ satisfying the relative longitudinal closing speed in Table 1 during the final translational phase. Figure 13 presents the norm of velocity tracking error which initially increases up to almost $0.145 \mathrm{~m} / \mathrm{s}$ because the closing transfer starts. It also increases to less than $0.0685 \mathrm{~m} / \mathrm{s}$ at the beginning of station-keeping and final translational maneuver at S3. Then, it finally converges to zero satisfying the relative longitudinal closing speed in Table 1. However, these increases can be allowed before the final translational maneuver. The norms of position and velocity tracking errors increase temporarily during the closing transfer and station-keeping at S3 in Figs. 10 and 13, and they can also be allowed because high-accuracy tracking results are not required at these phases. Finally, the chaser satisfies the minimum requirement for the final translational maneuver in Table 1 to enter the docking phase.

\subsection{Rotational maneuver result}

Figure 14 shows the chaser attitude trajectory with respect to the commanded trajectory, which is defined in the 


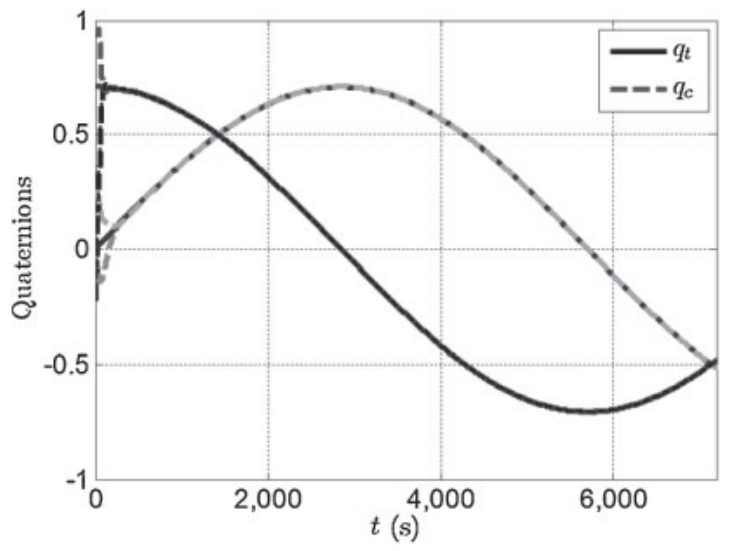

Fig. 14. Quaternions of the target and chaser.

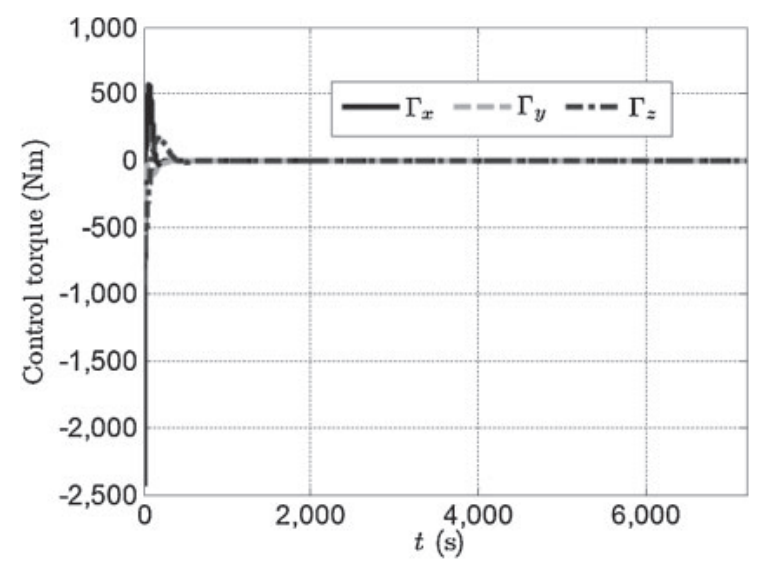

Fig. 15. Control torque.

inertial frame. As can be seen, the chaser is able to track the command precisely and quickly. The control torques are also initially large while the translational maneuver is performed simultaneously after which a smaller amount of control torque is required to maintain the desired attitude. Figure 15 shows the history of the control torques. A relatively large control torque is required initially to drive the chaser to the desired attitude quickly. Then, the control torque decreases rapidly after some oscillations to maintain the chaser at the desired attitude.

Figure 16 shows the chaser angular velocity. The chaser angular velocities track the fixed target angular velocity $\left[\begin{array}{lll}0 & 0.0011 & 0\end{array}\right]^{\mathrm{T}} \mathrm{rad} / \mathrm{s}$ which corresponds to an Earthpointing spacecraft. Figure 17 shows the Euler angles from the quaternion errors between the target and chaser using the SDRE control only and proposed control, respectively.

The Euler angles diverge when the SDRE control is used whereas they converge to zero when the proposed control is used in the presence of external disturbance torques and uncertain moment of inertia. Figure 17 obviously verifies that the proposed control is more effective than the conventional SDRE control in the presence of external disturbance torques and uncertain moment of inertia. In addition, the attitude tracking results in Figs. 14, 16 and 17 demonstrate that the minimum requirements for rotational maneuver in

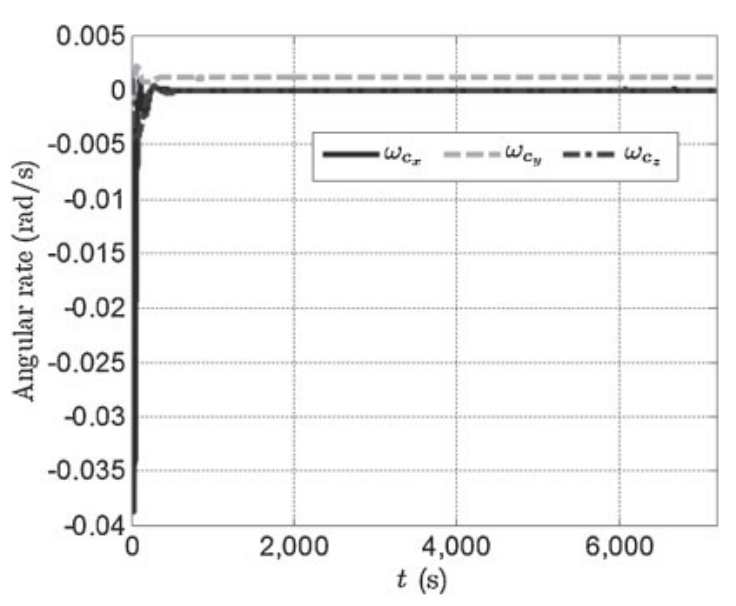

Fig. 16. Angular velocity of the chaser.

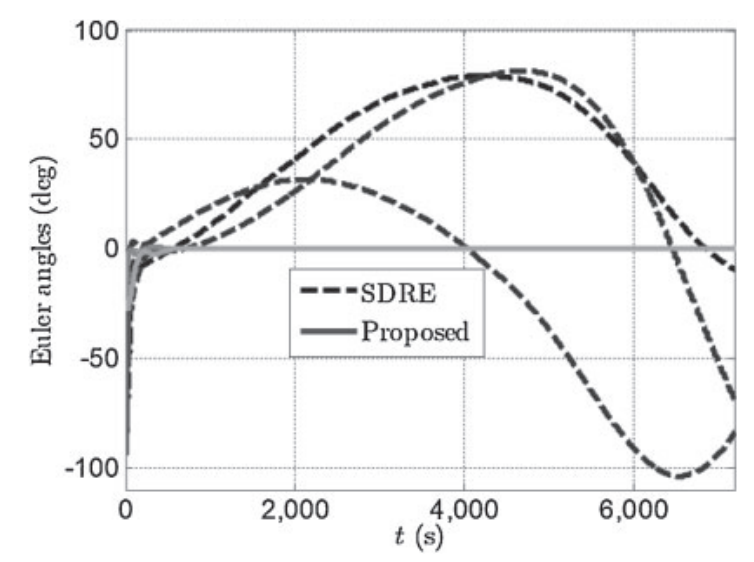

Fig. 17. Euler angles of the chaser.

Table 1 are successfully achieved.

\section{Conclusion}

This paper proposed a new control scheme based on a nonlinear output tracking and disturbance rejection in a closed-loop structure to apply for autonomous close-range rendezvous and docking of spacecraft. The control method was applied for the position and attitude tracking of a spacecraft in the presence of disturbances and the uncertainties of plant parameters. The control forces and control torques were simultaneously used to perform close-range rendezvous that requires both precise translational and rotational maneuvers. A 6 DOF simulation was utilized to demonstrate excellent tracking performance in the presence of external disturbances as well as the uncertainties of plant parameters. Compared with the conventional state-dependent Ricatti equation control method, the proposed control scheme yielded effective and robust tracking performance by rejecting disturbances using the state feedback control based on the state tracking errors. Simulation results also demonstrated that the proposed controller could successfully perform close-range rendezvous and achieve the minimum requirements to be reached at the docking phase. 


\section{References}

1) Fehse, W.: Automated Rendezvous and Docking of Spacecraft, First Ed., Cambridge University Press, New York, 2003.

2) Stansbery, D. T. and Cloutier, J. R.: Position and Attitude Control of a Spacecraft Using the State Dependent Riccati Equation Technique, Proceedings of the American Control Conference, IL, 2000, pp. 1867-1871.

3) Xin, M. and Balakrishnan, S. N.: Robust State Dependent Riccati Equation Based Spacecraft Attitude Control, The 40th AIAA Aerospace Sciences Meeting and Exhibit, Reno, NV, 2002.

4) Menon, P. K., Lam, T., Crawford, L. S. and Cheng, V. H. L.: RealTime Computational Methods for SDRE Nonlinear Control of Missiles, American Control Conference, AK, 2002, pp. 232-237.

5) Cimen, T.: Survey of State-Dependent Riccati Equation in Nonlinear Optimal Feedback Control Synthesis, J. Guid. Control Dynam., 35, 4 (2012), pp. 1025-1047.

6) Ghartermani, M. K., Khajehoddin, S. A., Jain, P. and Bakhshai, A.: Linear Quadratic Output Tracking and Disturbance Rejection, Int. J. Control, 84, 8 (2011), pp. 1442-1449.

7) Segal, S. and Gurfil, P.: Effect of Kinematic Rotation-Translation Coupling on Relative Spacecraft Translational Dynamics, J. Guid. Control Dynam., 32, 3 (2009), pp. 1045-1050.

8) Clohessy, W. H. and Wiltshire, R. S.: Terminal Guidance System for Satellite Rendezvous, J. Aerospace Sci., 27, 9 (1960), pp. 653-658.

9) Prussing, J. A. and Conway, B. A.: Orbital Mechanics, Oxford
University Press, New York, 1993.

10) Pinard, D., Reynaud, S., Delpy, P. and Strandmoe, S. E.: Accurate and Autonomous Navigation for the ATV, Aerospace Sci. Technol., 11, 6 (2007), pp. 490-498.

11) Farbrega, J., Frezet, M. and Gonnard, J.: ATV GNC during Rendezvous, Proceedings of the 3rd International Conference on Spacecraft Guidance, Navigation and Control Systems, ESTEC, Noordwijk, The Netherlands, 1996, pp. 18-21.

12) Schaub, H. and Junkins, J. L.: Analytical Mechanics of Aerospace Systems, American Institute of Aeronautics and Astronautics, New York, NY, 2003.

13) Lee, D. and Pernicka, H.: Optimal Control for Proximity Operations and Docking, Int. J. Aeronaut. Space Sci., 11, 3 (2010), pp. 179-193.

14) Vallado, D. A.: Fundmentals of Astrodynamics and Applications, Space Technology Library, Mircrocosm Press, El Segundo, California, 2004.

15) Cloutier, J. R. and Stansbery, D. T.: The Capabilities and Art of State-Dependent Riccati Equation-Based Design, Proceeding of the American Control Conference, AK, 2002, pp. 86-91.

16) Lee, D., Cochran, J. E. and No, T. S.: Robust Position and Attitude Control for Spacecraft Formation Flying, J. Aerospace Eng., 25, 3 (2012), pp. 436-447.

17) Nagata, T., Modi, V. J. and Matsuo, H.: Dynamics and Control of Flexible Multibody Systems Part II: Simulation Code and Parametric Studies with Nonlinear Control, Acta Astronautica, 49, 11 (2001), pp. 595-610. 